\title{
AIDS related Kaposi's sarcoma presenting as ulcerative colitis and complicated by toxic megacolon
}

\author{
B A BIGGS, S M CROWE, C R LUCAS, M RALSTON, I L THOMPSON, \\ AND K J HARDY
}

From the Fairfield Infectious Diseases Hospital, Fairfield, Victoria, Australia, St Vincent's Hospital, Sydney, and Austin Hospital, Melbourne, Australia

SUMmary Gastrointestinal Kaposi's sarcoma is a well described and usually asymptomatic manifestation of the acquired immune deficiency syndrome. We report a patient who had extensive colonic Kaposi's sarcoma and presented with an ulcerative colitis like illness. Total colectomy was subsequently required as an emergency procedure for toxic megacolon. The patient remains well on maintenance interferon therapy 21 months after surgery.

Kaposi's sarcoma is a well recognised manifestation of the acquired immune deficiency syndrome (AIDS) in homosexual men.' In the setting of AIDS the tumour follows a much more aggressive course than observed with immunocompetent patients. ${ }^{2}$ Usually there is widespread cutaneous involvement with a high incidence of gastrointestinal and visceral disease. Despite the presence of disseminated malignancy, however, these patients are often relatively well, and, in particular, gastrointestinal symptoms such as diarrhoea and rectal bleeding are unusual. ${ }^{3+}$

This is the case of a male homosexual who presented with apparent severe ulcerative colitis and who was found at laparotomy to have extensive colonic Kaposi's sarcoma. He subsequently survived two major operations; subtotal colectomy with ileostomy and an abdominoperineal resection. To our knowledge, it is the first case reported of severe gastrointestinal Kaposi's sarcoma as a manifestation of AIDS being successfully treated by surgery.

\section{Case report}

A 23 year old male homosexual presented to St Vincent's Hospital, Sydney, in October 1984 with a four month history of diarrhoea and weight loss of $5 \mathrm{~kg}$. During this time he had had 10-13 bowel actions

Address for correspondence: Dr B A Biggs. Fairfield Hospital, Yana Bend Road, Fairficld, Vic. Austratia 3078.

Received for publication 19 February 1987. per day and associated colicky abdominal pain. There was diffuse abdominal tenderness and enlargement of left cervical and axillary lymph nodes. No skin lesions were present nor was there a history of previous skin lesions. Antibodies to HTLV-III were present on both ELISA and Western blot assays. Faeces microscopy, including examination for Cryptosporidium oocysts, and culture for bacteria, mycobacteria and fungi were negative. Barium enema showed loss of haustral pattern with diffuse fine mucosal ulceration throughout the colon (Fig. 1). No plaque or nodular lesions were seen. A sigmoidoscopic biopsy showed moderately severe proctitis with a mixed acute and chronic inflammatory cell infiltrate, occasional crypt abscesses and moderate mucus cell depletion. No submucosa was present. A provisional diagnosis of ulcerative colitis was made.

In November 1984 he was started on salazopyrin and prednisolone $(30 \mathrm{mg})$ because of worsening diarrhoea and abdominal pain. This produced relief over the next two months and the dose of prednisolone was gradually reduced. At the end of January 1985 his condition deteriorated and he was readmitted to hospital. The dose of prednisolone was increased and this, combined with predsol enemas and rehydration, seemed to control the diarrhoea and rectal bleeding. Microbiological investigations were negative and sigmoidoscopy and biopsy were again consistent with ulcerative colitis. 


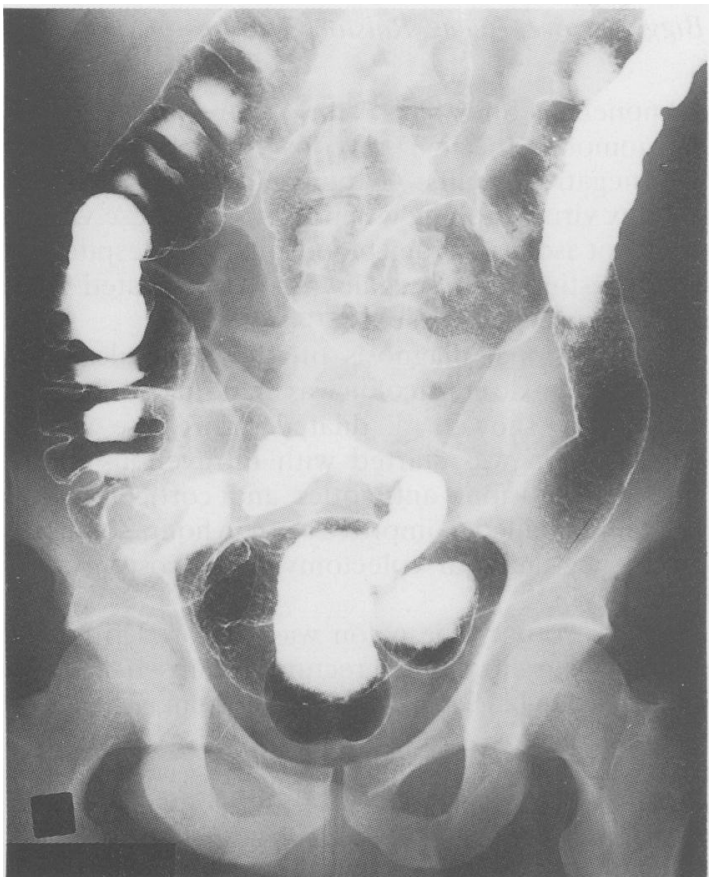

Fig. 1 Barium enema showing loss of the haustral pattern in the transverse, descending, and sigmoid colon. Finely granular mucosal pattern is present in these regions and is indicative of diffuse fine mucosal ulceration.
Two weeks after discharge and three months after initial presentation the patient developed several skin nodules behind his right ear. left thigh and over his shoulders and abdomen. A diagnosis of Kaposi's sarcoma was confirmed by skin biopsy. Gastroscopy revealed many sarcomatous lesions in the first and second parts of the duodenum. Sigmoidoscopic biopsy showed inflammatory changes as before, together with a proliferation of spindle cells arranged in interweaving fascicles infiltrating from submucosa into mucosa. Scattered, widely dilated, thin walled vascular channels, frequently of bizarre outline, extravasated red blood cells and haemosiderin were also present (Fig. 2). Immunoperoxidase staining using antiserum to Factor VIII related antigen showed positive staining of both the abnormal blood vessels and of the proliferating spindle cells. These appearances were characteristic of Kaposi's sarcoma.

Therapy was started with recombinant alpha interferon and vinblastine. At the end of March, however, the patient decided to move to his home city because of persistent ill health associated with chronic diarrhoea.

He presented to Fairfield Infectious Discases Hospital in April 1985, complaining of severe abdominal pain of several hours duration, fever and constipation. He appeared pale and distressed. The pulse was $120 / \mathrm{min}$, blood pressure $90 / 60 \mathrm{mmHg}$ and temperature $39 \cdot 2^{\circ} \mathrm{C}$. There were generalised

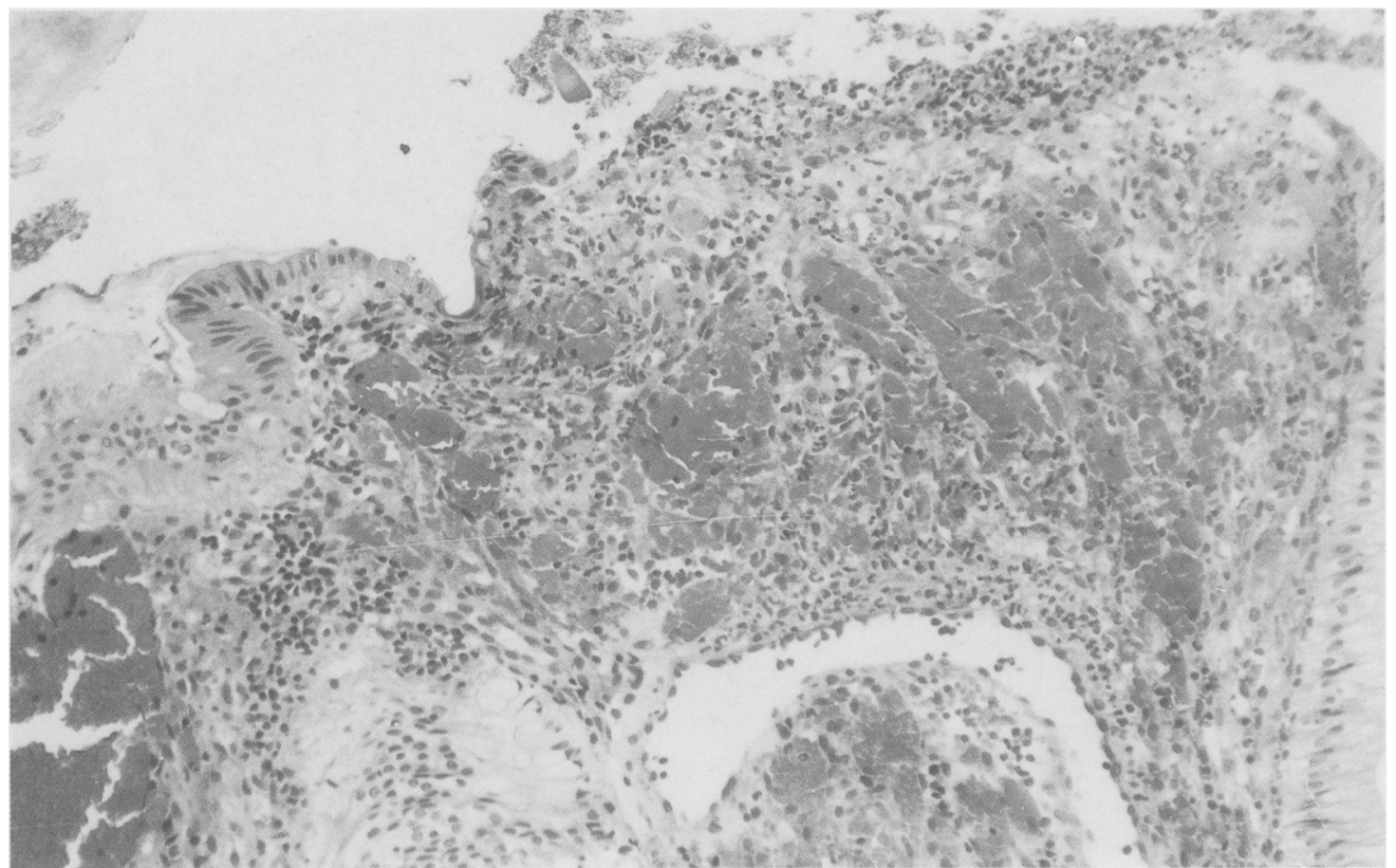

Fig. 2 Superficial mucosal erosion, with an underlying network of small, congested blood vessels. $H$ and $E$. 


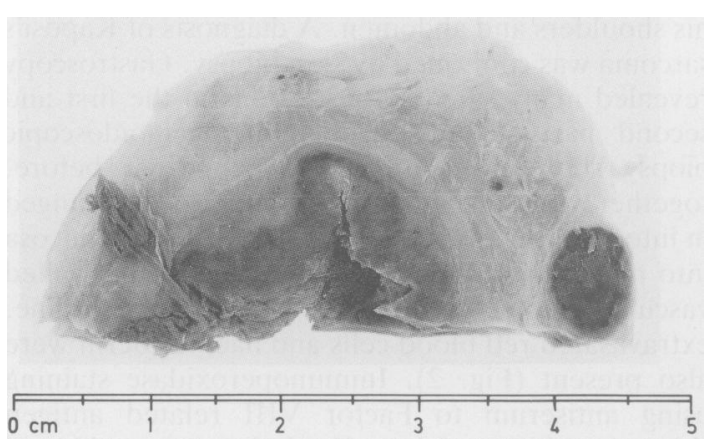

Fig. 3 Transverse section of the rectum showing the darkened area where there is infiltration of the mucosa and submucosa by Kaposi's sarcoma. Lymph node on the right side is expanded and largely replaced by tumour tissue.

Kaposi's sarcoma lesions involving skin, palate, and tonsils. The cervical, axillary and inguinal lymph nodes were enlarged. The abdomen was distended and on palpation there was general and rebound tenderness. Bowel sounds were noted to be absent. Faeces was examined by light and electron microscopy for parasites and viruses and cultured for bacteria (Campylobacter jejuni, Clostridium difficile,
Salmonella, Shigella, Vibrio, Aeromonas and Pleisiomonas species), mycobacteria and fungi, all with negative results. Cytomegalovirus and Herpes simplex virus serology was positive but these viruses were not isolated from blood or urine despite frequent testing. Cytomegalovirus was isolated from saliva on one occasion only.

A provisional diagnosis of fulminant ulcerative colitis with toxic megacolon was made and abdominal radiograph showed a dilated transverse colon. Resuscitation was started with intravenous fluids, blood transfusion, antibiotics and corticosteroids. The patient failed to improve over 36 hours so that an emergency subtotal colectomy and ileostomy was necessary.

At laparotomy the colon was dark red from the splenic flexure to the mid rectum, collapsed and with scattered jet black areas of up to $2 \mathrm{~cm}$. The distal rectum was grossly oedematous and the caecum and transverse colon were dilated. There was no evidence of disease in the small bowel, liver or spleen.

The postoperative period was uneventful. Alpha interferon and vinblastine therapy was started after two weeks when adequate wound healing had occurred. The patient remained well until the end of June 1985 when he required readmission to hospital

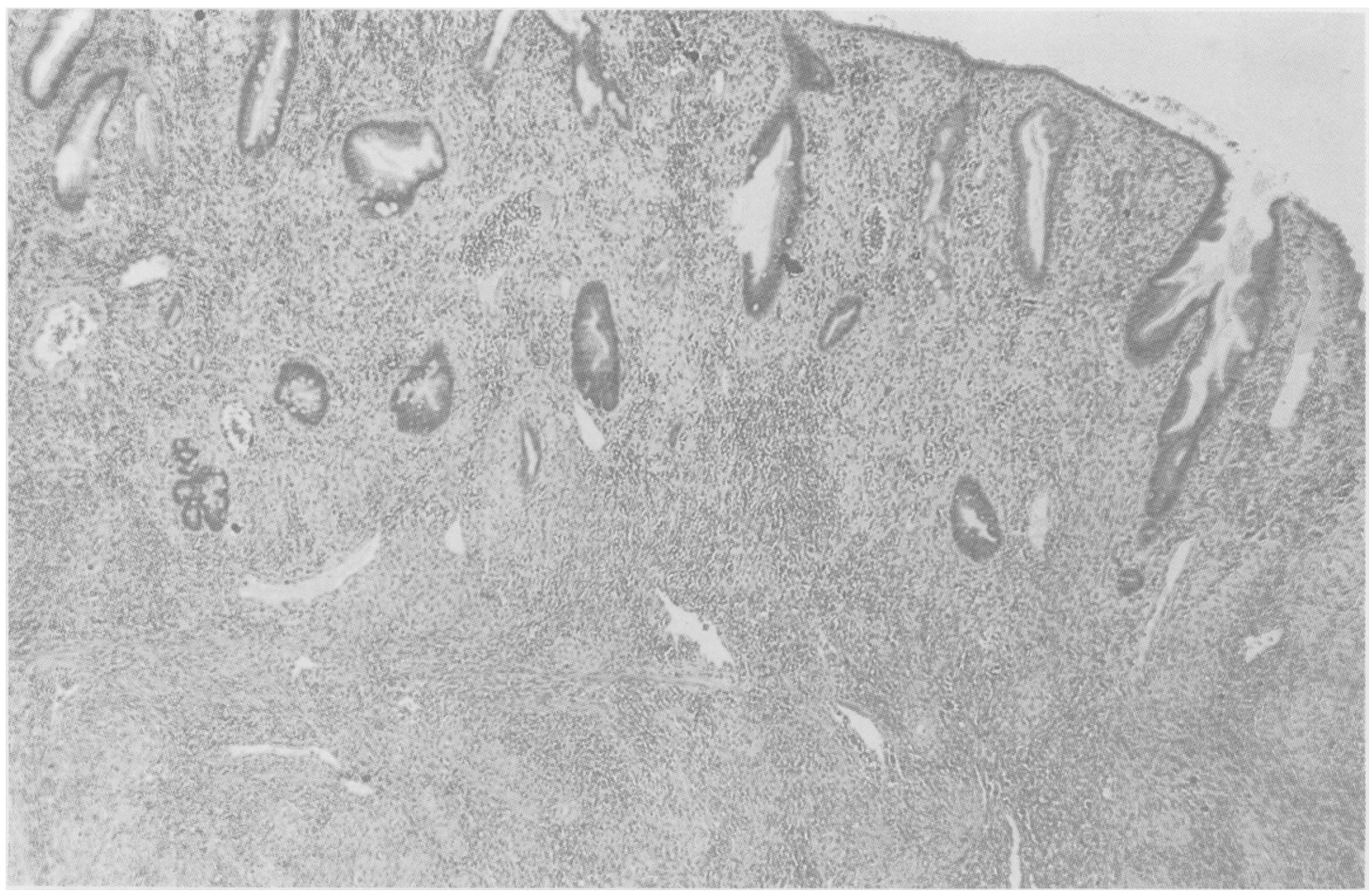

Fig. 4 Section of the mucosa showing extensive infiltration by Kaposi's sarcoma, with some loss of normal glandular elements. $H$ and $E$. 
because of worsening lower abdominal cramps and rectal discharge. Though an ileorectal anastomosis had been planned, these symptoms and the risk of Kaposi's sarcoma in the rectum made rectal excision the preferred treatment. An abdominoperineal resection was thus carried out. At laparotomy, the distal one third of the ileum was red and friable. The rectal stump was no longer oedematous but contained multiple dark areas, consistent with Kaposi's sarcoma (Fig. 3) which were not present at the previous laparotomy. Samples of rectal stump discharge and the rectal stump were again submitted for microbiological examination with negative results.

The pathology of the colon, rectum, and anal canal were similar: the mucosa of the caecum, ascending and proximal transverse colon was studded with multiple haemorrhagic nodules. More distally the mucosa was ulcerated (Fig. 4) and uniformly haemorrhagic with many small pseudopolyps. Histologically there was evidence of invasion of the bowel wall and pararectal lymph nodes by a solid and vasoformative malignant spindle cell tumour consistent with Kaposi's sarcoma (Fig. 5).

Twenty one months after surgery the patient remains well. The oral and cutaneous Kaposi's sarcoma lesions have disappeared with thrice weekly interferon therapy and his most recent helper $T$ cell count was within normal limits ( $T$ helper: $\mathrm{T}$ suppressor $=1 \cdot(03)$.

\section{Discussion}

The diagnosis of Kaposi's sarcoma of the bowel may be difficult to establish in the absence of skin and oral involvement. If the characteristic reddish nodules, plaques or macules are not evident endoscopically, or if they are obscured by features of inflammatory bowel disease, biopsy sampling error is very likely. In our patient the earlier mucosal biopsies failed to sample diagnostic tissue. A provisional diagnosis of ulcerative colitis was made on the basis of the mucosal inflammatory cell infiltrate, the crypt abscesses and mucus cell depletion, together with the barium enema features.

Kaposi's sarcoma of the bowel predominantly involves the submucosa, with later involvement of the mucosa and sometimes also of the deeper layer of the bowel wall. When considering this diagnosis, biopsies that include submucosa are mandatory. In our patient we believe that the inflammatory pathology present in the mucosa was secondary to the underlying submucosal Kaposi's sarcoma which only

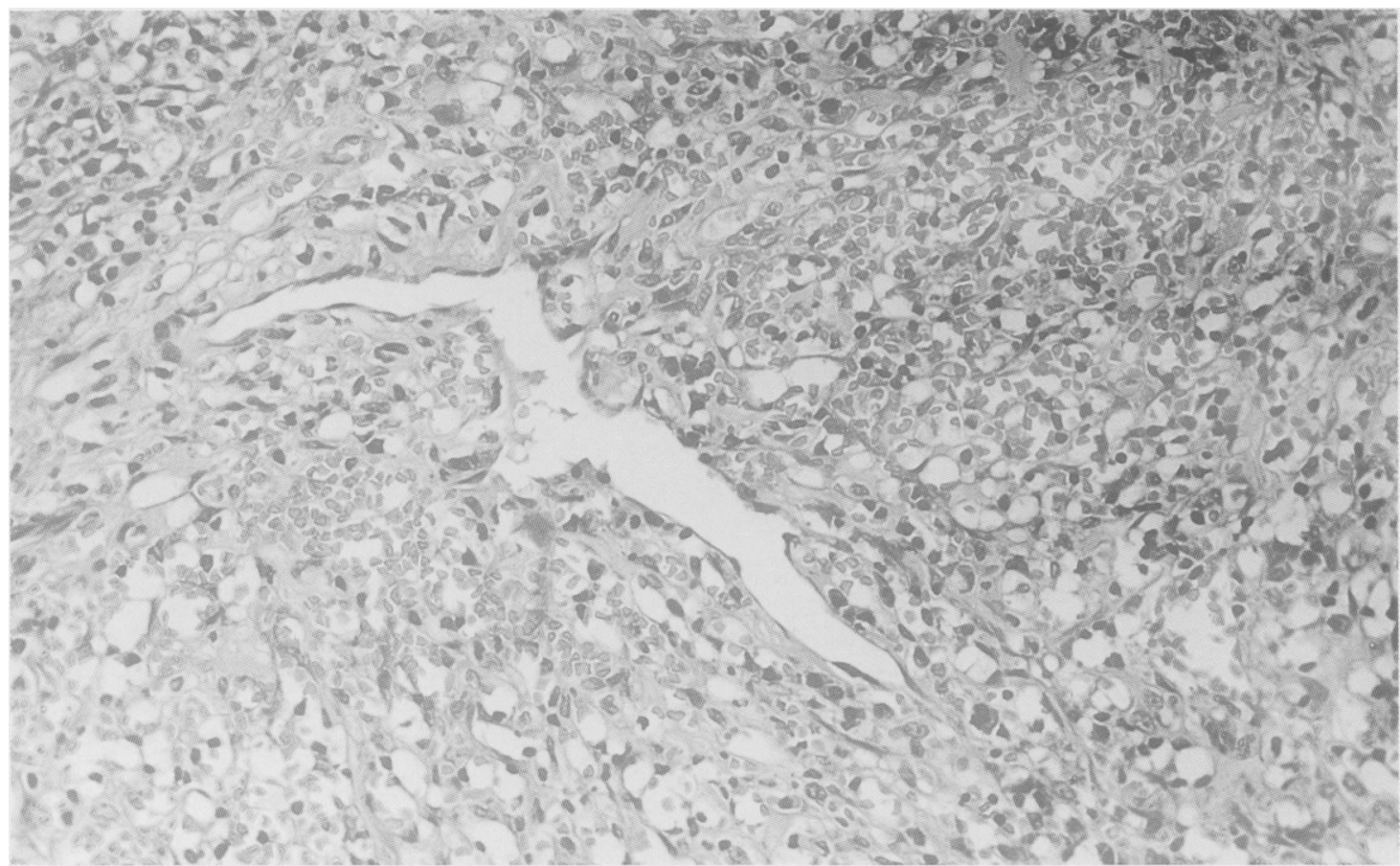

Fig. 5 Sarcomatous infiltrate, consisting of irregular thin walled vascular channels, small blood vessels, spindle cells and extravasated erythrocytes. $H$ and $E$. 
later infiltrated through the muscularis musocae into the mucosa. Before reaching this conclusion, a thorough effort was made to exclude the presence of a microbial pathogen which may have caused or aggravated the observed proctocolitis. Possibly, though not in the present case, immunoperoxidase staining for Factor VIII may sometimes permit earlier demonstration of infiltration of the mucosa by tumour. ${ }^{5}$

Radiological examination though helpful in demonstrating plaques and nodules will often fail to detect the more common macular lesions. Kaposi's sarcoma of the bowel presenting as apparent ulcerative colitis has recently been reported in an AIDS patient, with the death of the patient from bowel perforation 20 months after the onset of symptoms. There are also a few reports, ${ }^{7-9}$ antedating the AIDS era, describing the association of the two conditions. It is of interest that in two of them improvement occurred after corticosteroid administration. In our patient the high and prolonged doses of prednisolone given may have further depressed immune function and led to dissemination of gastrointestinal lesions and the development of cutaneous disease.

The satisfactory outcome of major surgery in the patient is noteworthy; the case appears to be the first reported instance of AIDS related Kaposi's sarcoma of the bowel complicated by toxic megacolon with survival, now 30 months since the onset of symptoms.
We thank Dr J D Wodak for performing the endoscopic examinations.

\section{References}

1 AIDS Surveillance Reports. Atlanta Georgia, USA: Centres for Disease Control, Week 11 November, 1985.

2 Friedman-Kien AE, Laubenstein LJ, Rubenstein P, et al. Disseminated Kaposi's sarcoma in homosexual men. Ann Intern Med 1982; 96: 693-700.

3 Friedman SL, Wright TL, Altman DF. Gastrointestinal Kaposi's sarcoma in patients with acquired immunodeficiency syndrome. Gastroenterology 1985; 89: 102-8.

4 Saltz RK, Kurtz RC, Lightdale CJ, et al. Gastrointestinal involvement in Kaposi’s sarcoma. Gastroenterology 1982; 82: 1168 .

5 Guarda LG, Silva EG, Ordonez NG, Smith JL. Factor VIII in Kaposi's sarcoma. Am J Clin Pathol 1981; 76: 197-200.

6 Weber JN, Carmichael DJ, Boylston A, et al. Kaposi's sarcoma of the bowel - presenting as apparent ulcerative colitis. Gut 1985; 26: 295-300.

7 Adlersberg R. Kaposi's sarcoma complicating ulcerative colitis: report of a case. Am J Clin Pathol 1970; 54: 143-6.

8 Gordon HW, Rywlin AM. Kaposi's sarcoma of the large intestine associated with ulcerative colitis. A hitherto unreported occurrence. Gastroenterology 1966; 50: 248-53.

9 Roth JA, Schell S, Panzarino S, Coronato A. Visceral Kaposi's sarcoma presenting as colitis. Am J Surg Pathol 1978; 2: 209-14. 